\title{
PRESENCIA CURRICULAR DE LA PSICOLOGÍA DE LA ACTIVIDAD FÍSICA Y DEL DEPORTE EN LOS PLANES DE ESTUDIO DE PREGRADO Y POSGRADO DE EDUCACIÓN FÍSICA VERSUS PSICOLOGÍA, EN MÉXICO
}

\author{
DR. PEDRO REYNAGA-ESTRADA
}

Laboratorio de Psicología de la Actividad Física y Deporte, Universidad de Guadalajara (Guadalajara - Jalisco - México) E-mail: preynagaestrada@yahoo.com.mx

\section{PSIC. ALEJANDRA YAZMIIN LANDEROS-RUBIO Laboratorio de Psicología de la Actividad Física y Deporte, Universidad de Guadalajara (Guadalajara - Jalisco - México) E-mail: aylr000 I@red.ujaen.es}

\author{
PSIC. TANIA LORENA ALONSO GÓMEZ \\ Laboratorio de Psicología de la Actividad Física y Deporte, \\ Universidad de Guadalajara (Guadalajara - Jalisco - México) \\ E-mail: tania_mayo28@hotmail.com
}

\section{PSIC. ANTONIO GARCÍA SANTANA}

Laboratorio de Psicología de la Actividad Física y Deporte, Universidad de Guadalajara (Guadalajara - Jalisco - México)

E-mail: Psic.antoniosantana@hotmail.com

\begin{abstract}
RESUMEN
El objetivo del estudio fue comparar la presencia curricular de las unidades de aprendizaje de Psicología de la Actividad Física en los planes de estudio de las licenciaturas (pregrado) y posgrado de Educación Física versus Psicología en las universidades que se ofertan en la República Mexicana. Estudio descriptivo y transversal que tuvo de universo 144 programas de estudio de psicología y 68 de educación fisica; encontrando que en los Posgrados de Psicología el peso porcentual es un poco mayor ( I 6.36) que en los Posgrados de Educación Física ( I 4. 33). Pero al unir pregrado y posgrado se triplica el peso porcentual de las unidades de aprendizaje en las disciplinas de Educación Física (3.13) a diferencia que en psicología (0.93). Estos resultados permiten concluir que, como eje de formación curricular, la psicología de la actividad física y del deporte tiene mayor presencia en Educación Física que en Psicología, lo que
\end{abstract}


significa la necesidad interdisciplinar y transdisicplinar de nuestro objeto de estudio; la unión e interconexión de la psicología y la actividad física y el deporte. Un problema y necesidad enfrentada fue la distinta nomenclatura de los programas de formación curricular tanto de los planes de estudio como de la unidad de aprendizaje, que pueden llevar a la necesidad nacional e internacional de acordar nombres, niveles, carga horaria y competencias (u objetivos) profesionales que pueden facilitar este tipo de análisis, pero sobretodo que faciliten el intercambio académico de estudiantes y profesores.

PALABRAS CLAVE: Psicología; deporte; Educación Física; evaluación educacional.

\section{INTRODUCCIÓN}

Para Ángelo e Gariglio (20 I 0), la formación docente en educación física refleja una orientación profesional que constituye a los saberes profesionales, generalmente identificados con una materia. Por ello quisimos conocer la presencia curricular de la Psicología de la Actividad Física y del Deporte en los planes de estudio de Educación física versus Psicología; utilizando como unidad de análisis a las materias, asignaturas, o ahora llamadas unidades de aprendizaje en el modelo vigente de competencias profesionales, en México.

En Brasil, existen diversos acercamientos al análisis curricular principalmente en Educación Física, pero de otras temáticas y con diferentes acercamientos metodológicos. Como ejemplos, Marin (200I) reflexiona sobre la necesidad de la formación profesional del lazer en educación física; Andrade Filho (200I) presenta un análisis sobre los cambios curriculares de los últimos años, las razones históricas que se vivieron y las formas en que se resolvieron ante las exigencias políticas internacionales; y Souza Neto et al. (2004) aportan el análisis legislativo del cambio curricular. Albuquerque, Casagrande y Taffarel (2007) también estudia la relación entre las concepciones epistemológicas y las prácticas pedagógicas. El estudio más cercano a nuestra investigación la presenta Molina Neto, Muller y Amaral (2003), quien también bajo un análisis histórico presenta el desarrollo de los posgrados de ciencias del movimiento en Brasil, de acuerdo a las temáticas de las asignaturas incluidas. Sin embargo, no realiza el análisis específico sobre nuestro objeto específico de la Psicología de la Actividad Física y del Deporte que se hizo en el presente estudio.

En Estados Unidos de Norteamérica se han hecho estudios curriculares sobre la formación en psicología de la actividad física y del deporte, para definir la orientación y formación profesional en el nivel del posgrado de los programas académicos de doctorado (DOSIL DÍAZ, 2008). Igualmente, también se presentan estudios sobre el origen de la formación profesional de los estudiantes que se incorporan en las maestrías y doctorados especializados en psicología de la actividad física y del deporte, lo cual no se vincula directamente con nuestro objeto de estudio. 
En México, como en la mayor parte del mundo, la inclusión de la Psicología del Deporte en el currículo de los planes de estudio se ha hecho por parte de las Licenciaturas en Educación Física y Cultura Física, así como en algunos posgrados específicos del país.

Lo anterior significa que contradictoriamente a lo posiblemente pensado, las aportaciones de la psicología no han sido desde esta disciplina hacia la cultura o educación física, sino desde el mismo seno de ésta última. Digamos que fue una generación in vitro.

Esto lo comprobamos en un estudio realizado en el año 2004 por Reynaga Estrada et al. (inédito), en un análisis de los planes de estudio de 92 instituciones universitarias públicas y privadas que ofrecen la licenciatura en Psicología en los diferentes estados de la República Mexicana, encontramos que solamente tres (3.26\%) de ellas incluían unidades de aprendizaje relacionadas a la Psicología del Deporte, lo cual nos da una idea de la desolación en este campo.

A nivel mundial, al menos en España, se observa un crecimiento de la incorporación de unidades de aprendizaje de psicología de la actividad física y del deporte en el curriculum formativo de las licenciaturas en psicología (CRUZ FELIU, 2002; DOSIL DÍAZ, 2008).

Como señala Dosil Díaz (2008), a pesar del creciente interés de los estudiantes de psicología y educación física hacia ésta área especíica, la inclusión de asignaturas de psicología de la actividad física se reserva por lo general al posgrado: diplomados, maestrías y doctorados.

Dosil Díaz (2008) señala que la formación específica en psicología de la actividad física y del deporte se puede realizar mediante cursos de doctorado y/o másteres/ cursos de especialización. Y realiza una revisión sobre la oferta de programas, planes de estudio e instituciones mediante las páginas web de las universidades. La ciencia actual no se entiende sin las nuevas tecnologías, la documentación y la información, que utilizadas como herramientas son imprescindibles del trabajo cotidiano. Esto es el antecedente más importante que justifica el presente trabajo. Sin embargo, el mencionado autor no realiza ni presenta ningún estudio como el presente. Solamente se presentan otras dimensiones de la formación en psicología de la actividad física y del deporte.

Coincidimos con Cruz Feliu (2002) en que la incorporación de la Psicología de la Actividad física y del Deporte en los planes de estudio debe incluir predominantemente un enfoque educativo y preventivo para que los estudiantes de la cultura y educación física puedan aplicarlo en el campo práctico, respetando las competencias y roles profesionales.

La formación del profesional de la educación física y del deporte es un área humana, y como tal es igual de compleja, por lo que requiere la colaboración de distintas ciencias y profesionales; y la psicología de la actividad física y del deporte 
confluye en ese objeto en común, que es la actividad física, la educación física y el deporte, para facilitar la labor de estos profesionales y la colaboración multi, inter y transdisciplinar (CANTÓN CHIRIVELLA, 2002; DOSIL DÍAZ, 2008; FENSTERSEIFER, 2006).

La multi e interdisciplinariedad es uno de los fundamentos de las ciencias del deporte. La necesidad de interacción de las diversas ciencias en el fenómeno de la educación física y el deporte son un hecho comprobado y el origen de la psicología en la cultura y educación física marca un reto importante en la colaboración multiprofesional.

Los planes de estudio requieren la incorporación competente de las distintas disciplinas y ciencias del deporte. Como señala Cruz Feliu (2002) y Cantón Chirivella (2002), esta tarea constituye un trabajo interdisciplinar entre psicólogos - del deporte y de la salud- y profesionales de la educación física, de la medicina y entrenadores; para recomendar el tipo de actividad o ejercicio físico más adecuado a cada grupo de población.

Los problemas del mundo actual requieren del trabajo multidisciplinar, pero si las profesiones y disciplinas intervienen aisladamente será difícil combatir fenómenos que requieren una intervención conjunta como la obesidad, el sedentarismo, y ciertas enfermedades crónico- degenerativas no infecto contagiosas.

Diversos autores explican que a nivel mundial, en países como Alemania, Estados Unidos, Inglaterra, Canadá y España, la psicología del deporte debe sus inicios a los profesionales de la educación física, quienes descubrieron la importancia de los factores psicológicos en el deporte. En gran medida la psicología fue atraída por los expertos en educación física y a partir de sus exigencias la psicología prestó atención al deporte y al ejercicio físico (CRUZ FELIU, 2002; DOSIL DÍAZ, 2008; GONZÁLEZ, 1997).

Algunos ejemplos de la inclusión de la psicología del deporte en la educación física datan desde 1921, con W. Schulte, N. Sippel y F. Giese, psicólogos de los Institutos de Educación Física de Leipzig y Berlín, en Alemania; quienes realizaron aportaciones experimentales y teóricas principalmente en el campo del aprendizaje motor. Los padres de la Psicología del Deporte en Rusia, Yuri Hanin, A.Z. Punin y Peter Rudik, también pertenecían al Instituto de Cultura Física, en el Instituto Central de Investigación Cientíica para el Estudio de la Educación Física en Moscú; y sus aportaciones se centraron en tiempo de reacción, personalidad y la influencia de otros factores psicológicos en diversos deportes (DOSIL DÍAZ, 2008; GONZÁLEZ, 1997).

El objetivo del estudio es observar (diagnosticar) el peso porcentual que se le otorga a las unidades de aprendizaje de psicología de la actividad física y del deporte con relación a la estructura total del plan de estudios que se ofertan en las 
Licenciaturas (pregrado) y posgrados de Educación (Cultura) Física y Psicología en las universidades de la República Mexicana, para comparar en cuál de éstas disciplinas (Educación física o psicología) se le otorga mayor importancia.

Se espera que este estudio sirva en el futuro para fundamentar la estructuración y justificación curricular de un posgrado en Psicología de la Actividad Física y del Deporte en la Universidad de Guadalajara, México.

Hasta el año 20 I I, en este estudio esperábamos encontrar en las distintas universidades en México mayor peso porcentual de las unidades de aprendizaje relacionadas con la Psicología de la Actividad Física y del Deporte en los planes de estudio de Psicología versus los planes de estudio de Educación Física y deporte. Esto debido a la afinidad profesional del campo de estudio.

\section{MATERIAL Y MÉTODOS}

Se realizó un estudio descriptivo, transversal, con fuentes electrónicas de internet de los planes de estudio ofertados por las distintas universidades mexicanas. Pretendiendo estudiar todos los planes de estudio en México, se encontraron 144 programas de estudio de psicología; y 68 programas de estudio de educación física, cultura física y/o ciencias del deporte en todos los Estados de la República Mexicana.

Wieder (2007) propone la organización del universo de la investigación educativa en los siguientes elementos:

I. Documentos sobre las prácticas educativas y culturales

2. Representaciones de las prácticas educativas y culturales

3. Instituciones

4. Aprendizaje

5. Enseñanza

6. Metodologías de trabajo

7. Sistemas e ideología de trabajo

8. Formación

9. Relación de la educación con la sociedad, el poder, la economía, etc.

De acuerdo a la información anterior de Wieder (2007) y Dosil Díaz (2008), son los planes y programas de estudio los documentos (electrónicos en nuestro caso) que manifiestan la importancia que se le otorga a una disciplina en el aprendizaje, la enseñanza y la formación académica y profesional. Por ello se realizó un check list de los Programas de estudio, ofrecidos y disponibles por vía internet que ofrecen las universidades públicas y particulares en las siguientes entidades de formación disciplinar y académica:

I) Licenciaturas y posgrados en Psicología 
2) Licenciaturas y posgrados en Educación Física, Cultura Física y Ciencias del Deporte.

Se excluyeron y/ o eliminaron todos aquellos programas de estudio inaccesibles por internet. Fuera que no apareciera la información de los programas y sus unidades de aprendizaje; o en los que aparecía la liga pero se tuvieron problemas de acceso.

En los planes de estudio de pregrado se tomaron en cuenta sólo aquellos que indicaran que se ofrecía el grado de licenciatura. Y para el posgrado, se incluyeron aquellos programas de estudio que indicaran que se trataba de diplomados, cursos, maestrías y doctorados, no importaba si eran instituciones públicas y/o privadas.

Debido a que es un estudio documental, sin trabajo con sujetos de estudio no implica ningún daño físico ni moral a personas. Además se respeta la confidencialidad de los datos de las universidades y planes de estudio; y tampoco se obtuvo apoyo financiero extraordinario, por lo que no requirió de aprobación de Comité de Investigación y/o Ética.

El procedimiento utilizado fue el siguiente:

I. Se elaboró una matriz para la base de datos en donde se incluían todos los Estados de la República Mexicana.

2. La búsqueda se repartió por Estados entre los colaboradores y se fueron escribiendo los nombres de las Universidades y los planes de estudio que ofertaban.

3. Se dividieron los planes de estudio de acuerdo a la disciplina de la que se trataba:

a) Psicología

b) Educación física, cultura física y/o cualquiera de las ciencias del deporte.

4. Posteriormente se capturó la información en hojas de cálculo del programa Excel para cada nivel de formación (pregrado y posgrado) por disciplina. Es decir:

a) Pregrado de Psicología

b) Posgrado de Psicología

c) Pregrado de Educación física y/o ciencias del deporte

d) Posgrado de Educación Física y/o ciencias del deporte.

5. En la hoja de Excel definida para cada nivel de formación, en una columna exclusiva se realizó el conteo de todas las unidades de aprendizaje que integraban cada plan de estudio; $y$ en otra columna se contaron las unidades de aprendizaje que tuvieran el nombre de Psicología de la Actividad Física y del Deporte, o nominación similar.

6. Con el conteo de las unidades de aprendizaje de los planes de estudio se obtuvo la distribución de frecuencias, sumando todas las unidades de aprendizaje que tuvieran el nombre de Psicología de la Actividad Física y/o del Deporte. 
Igualmente se sumaron en otra columna las demás unidades de aprendizaje. Para Moya (1 986, p. 89) esto es conocido como razón debido a que "una razón es una frecuencia relativa que relaciona la frecuencia de una categoría con la frecuencia absoluta de otra categoría, también se obtiene una razón cuando se relacionan las cifras absolutas de dos conjuntos diferentes". Las frecuencias totales obtenidas se refirieron a los niveles de formación por disciplina, mismas que constituyeron las categorías de análisis: distinguiendo dos grupos: a) las unidades de aprendizaje referidas a la Psicología de la Actividad Física y del Deporte; y b) todas aquellas unidades de aprendizaje diferentes según el nivel y disciplina en: Pregrado de Psicología; Posgrado de Psicología; Pregrado de Educación física y/o ciencias del deporte; Posgrado de Educación Física y/o ciencias del deporte.

7. Posteriormente se estandarizaron los grupos distintos utilizando proporciones. Según Levin (1979, p. 17) los datos de frecuencia permiten comparar distintos grupos, estandarizando las distribuciones mediante la proporción y el porcentaje. "Podemos convertir cualquier frecuencia en una proporción P, dividiendo el número de casos en cualquier categoría dada f por el número total de casos en una distribución N". O sea

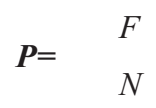

Donde $f$ es igual al número de unidades de aprendizaje con el nombre de Psicología de la Actividad física y del Deporte; y N es igual al número total de unidades de aprendizaje de los programas curriculares, fuesen de Educación Física o de Psicología, en posgrado y posgrado.

8. Una vez obtenida la frecuencia (Proporción) ésta se multiplicó por cien para obtener el tamaño relativo en términos del porcentaje. Mediante la siguiente fórmula:

$$
\%=(100) \quad \begin{aligned}
& F \\
& N
\end{aligned}
$$

9. Se calculó el porcentaje para poder comparar las unidades de aprendizaje de Psicología de la Actividad Física y del Deporte en contra de todas las demás unidades de aprendizaje de los planes de estudio de Psicología versus Educación Física y del Deporte.

I0. Por ello, en los resultados presentamos el porcentaje total por cada nivel de formación y por disciplina multiplicando la cantidad de unidades de aprendizaje de psicología de la actividad física y del deporte por 100 y dividiéndolo entre 
todas las demás unidades de aprendizaje, como puede observarse en las tablas presentadas en los siguientes resultados.

\section{RESULTADOS}

Se realizó un análisis de los planes de estudio de conformidad al procedimiento descrito previamente en la metodología y encontramos un total de 144 programas de estudio de psicología ofertadas por diferentes universidades de México, según se observa en la Tabla I. En dicha Tabla se puede observar que a pesar de que en el posgrado se encuentran menor cantidad de programas de estudio, y unidades de aprendizaje, el peso porcentual de las unidades de aprendizaje es mucho mayor con una distancia porcentual enorme ( $0.2 \%$ en pregrado y 16.36 en posgrado). Esto se debe a que los tres programas especializados en psicología de la actividad física y del deporte corresponden al posgrado, y a ellos corresponden la mayoría de las unidades de aprendizaje.

Respecto a los programas de cultura, educación física y ciencias del deporte se localizaron 68 programas de estudio de las diferentes universidades que son ofertadas en el país. De estas, al igual que en psicología, la mayor parte se concentran en el pregrado y contradictoriamente, aunque el porcentaje de las unidades de aprendizaje es mucho mayor en pregrado que en posgrado, las asignaturas de psicología de la actividad física y del deporte tienen cerca de siete (7) veces mayor porcentaje que en pregrado.

Comparando el peso porcentual con el que incluyen a la psicología de la actividad física y del deporte, se observa que si bien en los Posgrados de Psicología el porcentaje de su presencia es mayor ( 16.36 ) que en los Posgrados de Educación Física y ciencias del deporte ( I4.33) no existe mucha diferencia. En cambio, cuando sumamos el pregrado y el posgrado en las disciplinas y calculamos el porcentaje de las unidades de aprendizaje respecto al total de las unidades de aprendizaje, observamos que en la Educación Física y Ciencias del Deporte se incluyen más unidades de aprendizaje de Psicología de la Actividad física y del Deporte, lo que casi triplica su peso porcentual (0.93\% para psicología y 3. I 3\% para la educación física y las ciencias del deporte). 
Tabla I. Unidades de aprendizaje de Psicología de la Actividad física y del deporte presentes por nivel de formación en Psicología de los programas universitarios consultados

\begin{tabular}{ccccccc}
\hline \begin{tabular}{c} 
Nivel de formación en $\begin{array}{c}\text { Psicología } \\
\end{array}$ \\
\cline { 2 - 8 }
\end{tabular} & $\begin{array}{c}\text { Programas Universitarios } \\
\text { Consultados }\end{array}$ & \multicolumn{2}{c}{$\begin{array}{c}\text { Total de las Unidades } \\
\text { de Aprendizaje }\end{array}$} & $\begin{array}{c}\text { Unidades de Aprendizaje } \\
\text { de Psicología de la Actividad } \\
\text { Física y del Deporte }\end{array}$ \\
\hline Pregrado & $\mathrm{f}$ & $\%$ & $\mathrm{f}$ & $\%$ & $\mathrm{f}$ & $\%$ \\
Posgrado & 134 & $93.05 \%$ & 8480 & $95.65 \%$ & 20 & $0.2 \%$ \\
Total & 10 & $6.94 \%$ & 385 & $4.34 \%$ & 63 & $16.36 \%$ \\
\hline
\end{tabular}

Tabla 2. Distribución de las unidades de aprendizaje de psicología por nivel de formación en Cultura Física, Educación Física y/o ciencias del deporte con relación a los programas universitarios consultados

\begin{tabular}{ccccccc}
\hline $\begin{array}{c}\text { Nivel de formación } \\
\text { en Educación } \\
\text { Física y Deportes }\end{array}$ & $\begin{array}{c}\text { Programas } \\
\text { Universitarios } \\
\text { Consultados }\end{array}$ & $\begin{array}{c}\text { Total de las Unidades } \\
\text { de Aprendizaje }\end{array}$ & $\begin{array}{c}\text { Unidades de Aprendizaje } \\
\text { de Psicología de la } \\
\text { Actividad Física } \\
\text { y del Deporte }\end{array}$ \\
\cline { 2 - 7 } & $\mathrm{f}$ & $\%$ & $\mathrm{f}$ & $\%$ & $\mathrm{f}$ & $\%$ \\
\hline Pregrado & 51 & $75 \%$ & 2811 & $91 \%$ & 57 & $2.02 \%$ \\
Posgrado & 17 & $25 \%$ & 279 & $9 \%$ & 40 & $14.33 \%$ \\
Total & 68 & $100 \%$ & 3090 & $100 \%$ & 97 & $3.13 \%$ \\
\hline
\end{tabular}

\section{DISCUSIÓN}

Queda claro que una limitación del presente estudio es que las universidades y programas de estudio que no ofrecen su oferta académica por red electrónica (internet) quedaron excluidos. Posiblemente esta limitación quede superada si se establece contacto también con informantes clave de los estados de la República Mexicana en los que no se encontraron programas de estudio de las disciplinas investigadas en este acercamiento por internet.

En el presente estudio de investigación se encontró un problema recurrente: la diversidad de nominación de las unidades de aprendizaje relacionadas con la psicología de la actividad física y del deporte; de los programas de Educación Física, cultura física y/o ciencias del deporte. Wieder (2007) coincide y explica en primer lugar este problema y otros que se enfrentan en el campo de la Educación Física:

1. Diversidad conceptual de los términos

2. Variabilidad institucional en la selección de los saberes multidisciplinarios y científicos 
3. Dificultad de identificar las materias y sus contenidos, así como las profesiones que deben ocuparse de sus campos.

En este análisis de los programas y unidades de aprendizaje coincidimos plenamente con esta problemática. Las diversas universidades presentan una variación constante de las unidades de aprendizaje o saberes científicos; y por lo tanto de sus contenidos. Afortunadamente la formación general (licenciatura) en psicología tenía mayor homogeneidad en su nominación.

Para la uniformidad de la nominación de las unidades de formación en psicología de la actividad física y del deporte actualmente se tiene el reto y la exigencia de seguir el modelo de competencias para el aprendizaje. Esta es una excelente oportunidad para definir la nominación de las asignaturas; su adecuación al nivel de formación; al plan de estudios y la disciplina o sub disciplina que apoya; ya que, como señala Dosil Díaz (2008) "en estos momentos se esté replanteando la estructura y los contenidos formativos de grado como de posgrado" de acuerdo a la convergencia con el resto de países de la Unión Europea (acuerdo de Bolonia). Con esto se pretende mejorar la calidad educativa. Para De la Fuente Arias (2003) y Lafuente et al. (2007), el modelo de competencias profesionales es un elemento que actualmente está influyendo en el diseño de los nuevos currículo a nivel Europeo y Latinoamericano. La Unión Europea (UE) desde finales de 1990 inició un plan futuro en el sistema educativo con impacto internacional con base en dos hechos históricos conocidos como los objetivos de Lisboa y el proceso de Bolonia. Con esta perspectiva, el diseño curricular basado en competencias pretende responder a las exigencias del entorno empresarial competitivo, con formación de recursos humanos, habilidades y competencias educativas más prácticas; y con formas concretas de evaluación.

Para la construcción de los planes de estudio y de las unidades de aprendizaje (o unidades de competencia) con el modelo de competencias para el aprendizaje en pregrado y posgrado tanto de psicología de la actividad física y del deporte como de Psicología general o especializante; y de Educación Física y/o Ciencias del Deporte, se pueden consultar diferentes documentos. Por ejemplo: Gutiérrez-Rosado y Pallares-Parejo (2007); Ibánez-Bernal, (2007); Pallarés, (2007); Ramos-López (2003); Roe (2003); etcétera. Pero también es importante realizar un análisis histórico de las necesidades curriculares para, desde la disciplina de las ciencias del deporte contribuir a resolver los problemas reales que enfrentan nuestros países latinoamericanos, rescatando experiencias como las de Brasil y otras naciones.

Si la primera dificultad con el que se enfrentan las investigaciones en este campo es el concepto y definición de la educación física, entonces el primer elemento de la formación cientíica y profesional del licenciado en educación física 
debe ser la claridad de su objeto de estudio. Esa puede ser la primera competencia que domine el estudiante. En principio, se debe aclarar el campo y objeto de estudio y de trabajo científico y profesional de la educación física.

La definición de educación física, de acuerdo con Alurralde (en REYNAGA ESTRADA et al., 2007) la concibe como la ciencia de los lineamientos generales que determinan los contenidos y métodos para el desarrollo de la actividad física como proceso pedagógico organizado y vinculado al sistema educativo. Wieder (2007) coincide con Reynaga Estrada et al. (2007) en que el concepto de actividad física puede constituirse como el objeto de estudio de las diferentes disciplinas como educación física, cultura física y las ciencias del deporte. Y aunque el objeto de estudio puede ser el mismo, lo que cambia es el enfoque o visión disciplinar, que para el caso de la Educación Física es el proceso pedagógico. El debate sobre las concepciones, conceptos y objetos de estudios de las disciplinas y nominaciones se puede profundizar en otro documento o consultar la bibliografía referida en éste párrafo o en la bibliografía.

La definición del programa de estudio (Educación Física, cultura física o ciencias del deporte) debe ayudar a entender la competencia específica que requiere el profesional de la educación física con relación a la psicología de la actividad física y del deporte. Por ejemplo, no es lo mismo hablar de "psicología del deporte de alto rendimiento", que "psicología aplicada a la educación física escolar" o "psicología de la actividad física en educación básica". La dirección y el sentido de la formación son diferentes. Habrá que definir la relación de la (s) unidad (es) de aprendizaje con la disciplina y/o sub disciplina brindando la oportunidad de aportar al perfil profesional la formación adecuada.

Según Dosil Díaz (2008), lejos de considerar los resultados encontrados en este estudio como una "confrontación", o un "conflicto territorial" entre la psicología y la educación física, se puede afirmar que es una adecuada relación transdiscplinar, un matrimonio sano, que puede tener tantos hijos como necesidades se tengan en las diferentes disciplinas y en su nivel de formación. Más bien sería una ruptura de la frontera disciplinar y un ejemplo de la unión interdisciplinar entre la educación física y la psicología en un objeto común: la actividad física. En donde existe plena coincidencia con Dosil Díaz (2008) es que en los resultados de este estudio también se demuestra que la balanza (de la presencia de la psicología de la actividad física y del deporte) se inclina hacia las instituciones de educación física; y que todavía es difícil encontrar asignaturas de este tipo en la formación en psicología. Y esto refuerza la idea de que la psicología en educación física forma parte integral del movimiento humano y que como tal, se contempla como una disciplina que permite mejorar la actividad física o el resultado en un determinado deporte. Más aún se puede decir 
que la psicología aportó la visión teórica y la educación física (y ciencias del deporte) han aportado el contexto formativo.

\section{CONCLUSIONES Y PROPUESTAS}

I) El estudio permitió cumplir con nuestro objetivo propuesto de observar (diagnosticar) el peso porcentual que se le otorga a las unidades de aprendizaje de psicología de la actividad física y del deporte con relación a la estructura total del plan de estudios que se oferta en las Licenciaturas (pregrado) y posgrados de Educación Física versus los planes de estudio de Psicología en las universidades de la República Mexicana, para comparar en cuál de éstas disciplinas (Educación física o psicología) se le otorga mayor importancia. Con los resultados encontrados se observó que el peso porcentual de las unidades de aprendizaje de Psicología de la Actividad Física y del Deporte se presenta tres veces más en los planes de estudio de Educación Física, que en los planes de estudio de la formación en Psicología.

2) Con este estudio queda contrarrestada nuestra idea inicial de que en los planes de estudio de México encontraríamos más unidades de aprendizaje relacionadas con la Psicología de la Actividad Física y del Deporte en los planes de estudio de Psicología versus los planes de estudio de Educación Física y deporte. Los datos demostraron que la presencia curricular de la Psicología de la Actividad Física y del Deporte es mayor en los planes de estudio de Educación Física que en los de Psicología.

3) El presente estudio nos ayuda a fundamentar empíricamente la estructuración y justificación curricular de un posgrado en Psicología de la Actividad Física y del Deporte en la Universidad de Guadalajara, México; que junto con un futuro estudio de mercado profesional, estamos seguros hará posible su realización en poco tiempo, con relación a los siguientes numerales:

4) Existe el reto de homogeneizar la nominación del objeto de estudio: ciencias del deporte, ciencias del movimiento, cultura física, educación física y/o actividad física para conocer sus diferencias y conexiones y automáticamente aclarar la intervención profesional. Falta caracterizar el o los contextos ocupacionales en los que operan los profesionales de la actividad física y del deporte; por ejemplo: las necesidades de adherencia a las actividades física y deportivas para la salud psicológica, la prevalencia de empleo en la práctica independiente, la influencia de los gobiernos en la práctica profesional, la amplitud de las regulaciones (por ejemplo, las legales); la protección del título de Diplomado, Maestría y Doctorado. La posición relativa en la 
competencia con otros profesionales (Entrenadores, Licenciados en Cultura Física y del Deporte, Licenciados en Educación Física, médicos, trabajadores sociales, administradores); el poder de los colegios profesionales y de los sindicatos, etc. Lo anterior debe capacitar a este especialista para que realice actividades distintas en determinada región o país, de acuerdo a los factores antes mencionados. Pero que mantenga el perfil profesional abierto a la colaboración multidisciplinar. En virtud de que el Consejo Mexicano de Investigación Educativa agrupa en el área I I la temática en relación a la "Educación y valores" en los campos de educación física, deportiva, de la motricidad, somática, de la sexualidad, recreación y expresión corporal; Wieder (2007) propone que se llame "Cultura del movimiento, la corporeidad y educación física", y más sintéticamente se propone que se defina como cultura física; y ésta incluiría a la educación física como una disciplina más específica. Éste concepto debe ser un referente que nos permita unificar, nominar convencionalmente y agrupar a las disciplinas dedicadas a este campo profesional. Aunque se han propuesto y utilizado los conceptos de ciencias del deporte, ciencias del movimiento, etcétera; éstos no resuelven la problemática porque las instituciones utilizan cualquiera de ellos sin un criterio unificador. Si se lograra, serviría en principio para buscar y comparar si se trata del mismo campo de estudio científico y profesional. Desde esta problemática, incluso para otro estudio, sería importante determinar las nominaciones para los programas; pero también los nombres que se utilizan para las asignaturas; sus cargas horarias y contenidos de cada una de las unidades de aprendizaje relacionadas con la psicología de la actividad física y del deporte.

5) Todavía falta un gran desarrollo para que la psicología de la actividad física y del deporte se consolide no sólo en México, sino en el mundo. Se puede empezar por definir con claridad las disciplinas que se deben incluir en los planes de estudio (que a su vez pueden homogeneizar sus nombres, nomenclaturas, contenidos, créditos y cargas horarias, como se señaló previamente) a partir de su nivel de formación, impacto curricular vertical o transversal, competencias definidas, etcétera; que permita el intercambio académico desde las diferentes universidades del mundo con la selección de asignaturas, estudiantes y profesores. También falta una definición clara del área profesional; el reconocimiento y la regulación en el marco de la normativa legal; una presencia normalizada en los centros de trabajo relacionados con el deporte (clubes, equipos, federaciones, polideportivos, etc.) y una presencia social amplia que permita conocer ésta profesión, lo que puede ofrecer y lo que cabe esperar de su intervención (CANTÓN CHIRIVELLA, 2002). 
6) Otro tema pendiente es la evaluación con criterios de calidad internacional y educativa de los cursos de formación que ofrecen las diferentes instituciones, de los programas educativos; de las instituciones mismas, y de los profesores y estudiantes. La preocupación cotidiana recurrente de los profesionales en psicología de la actividad física y del deporte es la calidad de los cursos de formación y las escuelas que los ofertan; igualmente o todavía con mayor preocupación está la calidad de la práctica pseudo profesional de quienes se dicen expertos en el área. Ante estas dificultades, y por ser un área emergente, es necesario que se constituyan asociaciones y colegios de psicología de la actividad física y del deporte que regulen estas prácticas y cursos de formación. Aunque también está el papel del Estado. Principalmente las autoridades universitarias en el caso de México, que son quienes regulan la incorporación y validez oficial de las instituciones. O muy particularmente la Secretaría de Educación (entidad federal responsable de estos asuntos) que tiene la facultad de verificar y regular la calidad de toda la formación educativa formal de las instituciones públicas y particulares en México.

\section{A presença da disciplina psicologia da atividade física e do esporte nos currículos de graduação e de pós-graduação em Educação Física e em Psicologia, em universidades mexicanas}

RESUMO: O presente estudo teve como objetivo comparar o peso percentual das unidades de aprendizagem de psicologia da atividade física na estrutura curricular para a formação profissional inicial e de pós-graduação nos currículos de Educação Física versus Psicologia, em universidades mexicanas. De caráter descritivo e transversal, e tendo como universo I 44 currículos de Psicologia e 68 de Educação Física, o estudo mostrou que nos Programas de pós-graduação em Psicologia o peso percentual é um pouco maior ( 16.36 ) que nos Programas de Pós-graduação em Educação Física (14.33). Entretanto, ao considerarmos graduação e pós-graduação conjuntamente, o peso percentual das unidades de aprendizagem é três vezes superior nas disicplinas de Educação Física (3. 13) em relação às de Psicologia (0.93). PALAVRAS-CHAVE: Psicologia; esporte; Educação Física; avaliação educacional.

\section{Curriculum Presence of Physical Activity and Sport Psychology in Undergrade and Graduate Physycal Education versus Psychology Curriculum, in Mexican Universities}

ABSTRACT: The aim of this study was to compare the percentage of Learning Units in Psychology of Physical Activity in the curriculum structure for the training of the degree (undergraduate) and graduate curricula in Physical Education versus Psychology offered in universities in Mexico. 
This study was descriptive and cross-sectional, it had an universe of I 44 programs of study in Psychology and 68 in Physical Education. We found that percentage in graduate programs of Psychology is slightly higher (16.36) than Graduate programs of Physical Education (1 4.33). But by combining Undergraduate and Graduate, the percentage of Learning Units in the disciplines of Physical Education (3.13) triples the percentaje weight in Psychology (0.93). These results allow the conclusion that, as the axis of training curriculum, Psychology of Physical Activity and Sport has a greater presence in Physical Education than in Psychology, which means the need interdisciplinary and transdisciplinary of our object of study; the connection and interconnection of the Psychology and the study of Physical Activity and Sport. A problem found during this study which needs to be faced was the nomenclature of the different training programs from both the curriculum plans of study and the learning units, which can lead to national and international agreements on names, levels, load time and professional competences (or targets) to provide this type of analysis, but especially to facilitate academic exchange programs of students and teachers.

KEYWORDS: Psychology; Sport; Physical Education; Educational Assessment.

\section{REFERENCIAS}

AlBUQUERQUE, J. O.; CASAGRANDE, N.; TAFFAREL, C. N. Z. A prática pedagógica da Educação Física no MST possibilidades de articulação entre teoría pedagógica, teoría do conhecimento e projeto histórico. Revista Brasileira do Ciências do Esporte, Campinas, v. 8, n. 2, p. $121-140,2007$.

CANTÓN CHIRIVELLA, E. El área profesional del la psicología del deporte. In: DOSIL DÍAZ, J. (Ed.). El psicólogo del deporte: asesoramiento e intervención. Madrid: Editorial Síntesis, 2002. p. 39-50.

CRUZ FELIU, J. Aplicaciones de la psicología aplicada al deporte. In: DOSIL DÍAZ, J. (Ed.). EI psicólogo del deporte: asesoramiento e intervención. Madrid: Editorial Síntesis, 2002. p. 23-38.

DE LA FUENTE ARIAS, J. ¿Por qué los alumnos no construyen un conocimiento psicológico académico y profesional integrado?: reflexiones para una investigación necesaria. Papeles del Psicólogo, Madrid, n. 86, p. 34-4I, 2003.

DOSIL DÍAZ, J. (Ed.). El psicólogo del deporte: asesoramiento e intervención. Madrid: Editorial Síntesis, 2002.

- Psicología de la actividad física y del deporte. 2. ed. Madrid: McGraw Hill: Interamericana de España, 2008.

FENSTERSEIFER, P. E. Corporeidade e formação do profissional na área da saúde. Revista Brasileira de Ciências do Esporte, Campinas, v. 27, n. 3, p. 93-102, 2006.

FIGUEIREDO DE ANDRADE FILHO, N. F. Formação profissional em Educação Física brasileira: uma súmula da discussão dos anos de 1996 a 2000. Revista Brasileira de Ciências do Esporte, Campinas, v. 22, n. 3, p. 23-37, 2001. 
GARCÍA UCHA, F. E. Formación y psicología del deporte profesional en Cuba. Revista de Actualidad de la Psicología del Deporte, v. I, n. 5, 2002.

GARIGLIO, J. A. O papel da formação inicial no processo de constitução da identidade profissional de professores de educação física. Revista Brasileira de Ciências do Esporte, Campinas, v. 32, n. 2/4, p. II-28, 2010.

GONZÁLEZ, L. Psicología del deporte. Madrid: Biblioteca Nueva, 1997.

GUTIÉRREZ ROSADO, T.; PALLARES-PAREJO, S. La titulación de psicología en España: primeras fases del proceso de adaptación al espacio europeo de enseñanza superior. Revista de Educación y Desarrollo, n. 6, p. 35-44, 2007.

IBÁNEZ BERNAL, C. Diseño curricular basado en competencias profesionales: una propuesta desde la psicología interconductual. Revista de Educación y Desarrollo, n. 6, p. 46-52, 2007.

LAFUENTE, J. V. et al. El diseño curricular por competencias en educación médica: impacto en la formación profesional. Educación Médica, v. I0, n. 2, p. 86-92, 2007.

LEVIN, J. Fundamentos de estadística en la investigación social. México: Harla, 1979.

MARIN, E. C. Currículo e formação profissional do lazer. Revista Brasileira de Ciências do Esporte, Campinas, v. 23, n. I, p. 123-130, 2001.

MOLINA NETO, V.; MULLER, M. A.; AMARAL, L. O programa de pós-graduação em ciências do movimento humano da ESEF/UFRGS: a visão dos estudantes sobre o seu processo de formação profissional. Revista Brasileira do Ciências do Esporte, Campinas, v. 24, n. 2, p. 75-96, 2003.

MORÁN DOSTA, C. Formación y psicología del deporte profesional en México. Revista de Actualidad de la Psicología del Deporte, v. I, n. 5, 2002.

MOYA, L. Introducción a la estadística de la salud: curso básico para estudiantes de ciencias de la salud. Costa Rica: Editorial de la Universidad de Costa Rica, 1986.

PALLARÈS, S. Evaluación de competencias de los estudiantes del master en gestión de los RRHH a través de un assessment center. Revista de Educación y Desarrollo, n. 6, p._55-6I , 2007.

RAMOS LÓPEZ, J. Retos actuales de la formación en psicología: análisis de las temáticas abordadas en los congresos nacionales sobre enseñanza de la psicología. Papeles del Psicólogo, n. 86, 2003.

REYNAGA ESTRADA, P. et al. El debate necesario entre la Educación Física, la cultura física y la actividad física: una reflexión para la formación profesional del estudiante. Revista de Educación y Desarrollo, n. 7, out./dec. 2007. Disponível em: http://www.cucs.udg.mx/ revistas/edu_desarrollo/anteriores/7/007_Reynaga.pdf.

ROE, R. ¿Qué hace competente a un psicólogo? Papeles del Psicólogo, n. 86, 2003. 
SOUZA-NETO, S. et al. A formação do profissional de Educação Física no Brasil: uma história sob a perspectiva da legislação federal no século XX. Revista Brasileira de Ciências do Esporte, Campinas, v. 25, n. 2, p. $113-128,2004$.

VÁZQUEZ, O.; RODRÍGUEZ-MARTíNEZ, P. Perspectivas de desarrollo para la psicología aplicada al deporte. Instituto Tecnológico y de Estudios Superiores de Occidente (ITESO). Disponível em: http:/www.efsi.iteso.mx/zona\%areas\%psicologia\%20deporte/articulos... Acesso em: 8 nov. 2004.

WIEDER, R. E. (Ed.). Corporeidad, movimiento y Educación Física: estudios conceptuales. México: Consejo Mexicano de Investigación Educativa, 2007. v. I .

Recebido em: 6 fev. 2012 Aprovado em: 16 abr. 2013

Endereço para correspondência:

Pedro Reynaga-Estrada

Circuito Hacienda del Carmen Oriente num. 25. Col. Hacienda Real, $2^{a}$. Sección, Tonalá, Jalisco. México. C.P. 45428. Teléfono y fax: (005233) 35624I 43 
